\title{
Working Conditions and Dentists' Attitude Towards Proprioceptive Derivation
}

\author{
Montakarn Chaikumarn \\ Department of Human Work Sciences, Luleå University of Technology, Luleå, Sweden
}

Proprioceptive derivation (Pd), a new method of organising a dentist workstation as well as a working procedure, was introduced to Thailand. The aim of this study was to assess the working conditions and the attitude to Pd among experienced users. Questionnaires were distributed among 12 dentists. The results showed that all dentists chose to work in a sitting posture and mostly worked without breaks between patients. They spent less time on dental examination and crown and bridge therapy tasks. Solving problems in patients with physical limitations resulted in a low stress level. Seven dentists (58.3\%) always used Pd and liked it. Five dentists (41.7\%) sometimes used $P d$, with 3 of them liking it. Only 2 dentists, who sometimes used $P d$, did not like it because it could not cover all dental tasks and treatment, and it was difficult and complex.

dentist working conditions attitudes proprioceptive derivation

\section{INTRODUCTION}

Dental work includes a wide range of physical hazards. Musculoskeletal disorders are one obvious hazard. They may be caused by exposure to high precision work with long-lasting static loads in the cervical and shoulder regions [1]. Moreover, dentistry is an occupation with high psychological demands and with other ergonomics risk factors, which require effective ergonomics interventions to solve those problems $[1,2]$. According to Pollack [3] the key aim of ergonomics for dentists is to allow them to achieve optimum access, visibility, comfort and control in clinical work.

The concept of ergonomics was introduced into dentistry in order to improve the dental profession's working conditions; the work concepts included sit-down and four-handed dentistry [4]. An American dentist, Dr. Daryl Beach, developed a new concept for dental practice. It focuses on the positions, movements, contacts and comfort that dentists can sense with their bodies [5]. This concept is widely known as proprioceptive derivation (Pd). However, when the concept was first introduced, it was also identified as system or performance logic. In the Pd concept the adjustable conventional dental equipment and the work process are causative factors behind the high prevalence of musculoskeletal discomfort in dentists. Therefore, instead of a tilted dental chair and an adjustable lamp, Pd introduces equipment with minimum adjustability. The patient lies horizontally during treatment, and the dentist consistently works in a full upright alert seated posture. The dentist's upright posture is considered to provide the best control of the fine stabilized finger movements required when operating in the mouth. By stabilizing the position of the mouth, the dentist and the assistant are able to easily reach necessary equipment and materials, they can work more accurately, more efficiently, and with less physical and mental wear and tear on both the patient and the dentist [6].

In this concept, proprioception means "a sense or perception, usually at a subconscious level, of the movements and position of the body and especially its limbs, independent of vision; this sense is gained primarily from input from sensory

The author would like to acknowledge all participants in this study as well as the Dean and staff of the Faculty of Dentistry, Thammasat University, Thailand. She also thanks Miss Nuttika Nakphet, for preparing the questionnaires and for other support in Thailand.

Correspondence and requests for offprints should be sent to Montakarn Chaikumarn, Department of Human Work Sciences, Luleå University of Technology, SE-97187 Luleå, Sweden. E-mail: <chmo@arb.luth.se>. 
nerve terminals in muscles and tendons (muscle spindles) and the fibrous capsule of joints combined with input from the vestibular apparatus" [7]. In other words, the concept of Pd allows the dentist to use proprioceptive self-awareness to determine the most efficient, stress-free process of performing dental procedures $[8,9]$.

When dentists learn $\mathrm{Pd}$, starting with the training period, the dentist is neither told nor shown how to sit, how to position the patient, or how to maintain the relations in the dental process (such as height of the supportive system, position of the dental instrument tray). Instead, dentists individually remember these setting via their proprioception. The determination of the dentist's posture, patient positioning, and the dental process are based on the five movements along with the ten-step protocol derived through the skilled practice of Pd [8].

\subsection{Five Movements}

During dental treatment based on Pd, dentists focus on five movements related to dentist

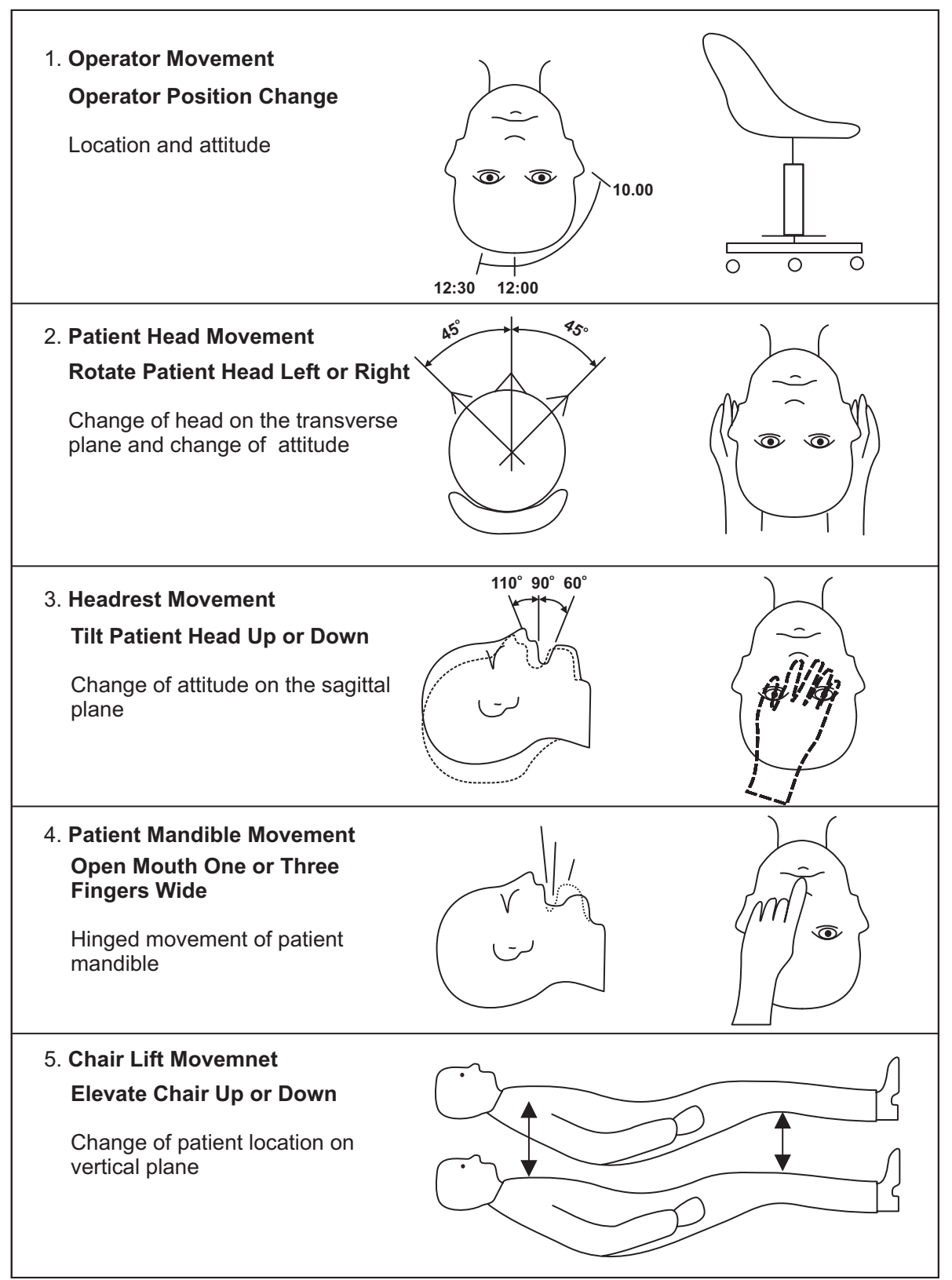

Figure 1. Five movements in Proprioceptive Derivation [8] (p. 292). 
performance, posture and patient's position (Figure 1):

1. Dentist movement around the patient's head in a clockwise or counter-clockwise direction;

2. Patient head movement by rotation to the left or right;

3. Patient head tilt upward or downward;

4. Patient mandible movement: minimising or maximising the mouth opening of patient;

5. Elevation of the patient's support by moving the supportive system upward or downward [8] (p. 291).

\subsection{Ten-Step Protocol}

Dentists are additionally given a ten-step protocol guide to optimal perception and control of dentist performance:

1. Establish appropriate inter-maxillary opening;

2. Grasp instrument or item to be used with thumb and index finger;

3. Place instrument or item to place to task site;

4. Stabilize instrument or item with middle finger on task site or as proximal as possible;

5. Check posture to determine whether steps 1-4 have compromised (to cause impairment) posture. If not, then process directly to step 6 . If posture is compromised, correct it by rotating patient's head right or left and by dentist's movement clockwise or counterclockwise;

6. Check vector of force application (axis of instrument or item) to assure alignment with mid-sagittal plane. If adjustment is necessary, correct by rotati ng patient's head or dentist's movement;

7. Plan to move instrument or item from distant point to near point on task site;

8. Establish eye-to-task sight line with direct or indirect view as appropriate;
9. Stimulate performance to ensure optimal performance;

10. Perform act to achieve the planned outcome [8] (p. 293).

$\mathrm{Pd}$ is also combined with a training program called SATV (Skill, Acquisition, Training and Verification), which helps dentists in gaining self-derived experience. The SATV system is divided into skill acquisition, skill transfer, and skill verification phases [6].

In the skill acquisition phase, dentists use models for training. Body positions and setting requirements that are compatible with the highest imaginable level of clinical performance are recorded. These conditions are considered to minimize physical stress during dental treatment. The derivations are then used to adjust the SATV clinical setting to the dentist's unique body dimensions for optimal delivery of care.

The skill transfer phase emphasizes that the acquired basic skills may be applied to clinical procedures such as oral examination, extraction, anaesthesia, tooth cavity restoration, root canal treatment and preparation for crowns and bridges.

Skill verification - by means of multimedia such as a camera, digital video recordings or data forms, and standardized simulated pathologies - of skill acquisition and transfer is used throughout the system.

Terui, Iwao, and Taniguchi [10] reported that, by using SATV, the concept of Pd can give dentists many benefits:

1. It establishes and maintains optimum finger control for precision work;

2. It minimises distraction from the care receiver (patient);

3. It helps to keep the dentist's spine healthy;

4. It maintains consistent accuracy, and minimizes treatment time by eliminate unnecessary acts;

5. It establishes a basis of infection control by minimizing the number of fingerinstrument contacts (p. 244). 
Pd has been used in many countries, such as Japan, North America and some countries in Europe. In Thailand, one dental school implemented $\mathrm{Pd}$ at the beginning of its foundation. The aim of using Pd is to improve dentists' health and performance, increase productivity and the quality of dental care [5].

Even though Pd has been used in many countries for many years, it is still considered as a new concept in Thai dentistry. There are few studies on how it could improve work performance or reduce musculoskeletal symptoms as Pd is claimed to do. For that reason, it is important to investigate working conditions and the dentists' attitude towards Pd, which were the aims of this study. The results - information necessary to make decisions before introducing any changes in dental clinics in relation to this concept - could benefit other dentists and dental schools. Further, the results could contribute to ergonomists' concern regarding dentists' workstation organization and the working process as well.

\section{PARTICIPANTS AND METHODS}

Twelve dentists (4 males and 8 females) participated in the study. They all worked as dentists and university lecturers. They all knew and used Pd. Their working experience of Pd ranged from 8 to 36 months. A two-part self-administered questionnaire was distributed to all participants.

Part 1 concerned individual characteristics (age, gender, handedness, level of education and years in profession) and working conditions (working hours, number of patients per day, working posture, working time, working technique and breaks between cases). They were asked about the working time spent on five main dental work tasks: dental examination, teeth cleaning, dental filling therapy, preparation for crowns and bridges, and tooth extraction and working situations causing stress. The dentists were asked to rate stress caused by each working situation on a 6-point rating scale (from 1-no stress at all to 6-very high degree of stress).

In part 2 of the questionnaire the dentists were additionally asked how often they used Pd, what

TABLE 1. Characteristics of Dentists $(n=12)$

\begin{tabular}{|c|c|}
\hline Characteristics & Value \\
\hline Age in years $(M \pm S D)$ & $29.2 \pm 8.7$ \\
\hline \multicolumn{2}{|l|}{ Gender } \\
\hline Male & 4 \\
\hline Female & 8 \\
\hline \multicolumn{2}{|l|}{ Handedness } \\
\hline Right-handed & 12 \\
\hline Left-handed & 0 \\
\hline Ambidextrous & 0 \\
\hline \multicolumn{2}{|l|}{ Education } \\
\hline DDS & 10 \\
\hline Master's/Postgraduate degree ${ }^{1}$ & 1 \\
\hline Ph.D. & 1 \\
\hline Years in profession $(M \pm S D)$ & $8.07 \pm 5.02$ \\
\hline $\begin{array}{l}\text { Working hours in clinic per day } \\
\qquad(M \pm S D)\end{array}$ & $5.42 \pm 1.16$ \\
\hline $\begin{array}{l}\text { Number of patients per day per } \\
\text { dentist }\end{array}$ & 8.5 \\
\hline
\end{tabular}

their attitude to Pd was and why.

\section{RESULTS}

Table 1 shows the participants' characteristics.

\subsection{Working Posture}

For all 12 dentists sitting was the only working posture in the clinic.

\subsection{Working Techniques}

All 12 dentists used the 4-handed technique: they always had dental assistants when they gave dental care to patients. 
TABLE 2. Time Spent on Each Dental Task

\begin{tabular}{lcc}
\hline \multirow{2}{*}{ Dental Task } & \multicolumn{2}{c}{ Duration (min) } \\
\cline { 2 - 3 } & $\boldsymbol{M} \pm \boldsymbol{S D}$ & Range \\
\hline Dental examination & $9.0 \pm 7.7$ & $3-30$ \\
Teeth cleaning & $24.1 \pm 5.9$ & $15-30$ \\
Dental filling therapy & $24.6 \pm 5.2$ & $20-30$ \\
Crown and bridge therapy & $42.0 \pm 21.0$ & $20-60$ \\
Tooth extraction & $16.0 \pm 6.1$ & $10-30$ \\
\hline
\end{tabular}

\subsection{Working Time Spent on Each Dental Work Task}

The dentists reported how much time, on average, they spent on each task. The result is presented in Table 2 .

\subsection{Breaks Between Patients}

Only five dentists (41.7\%) reported that they had breaks between patients. Their average duration was $5 \mathrm{~min}$.

TABLE 3. Dentists' Feelings on Work $(n=12)$

\begin{tabular}{lc}
\hline Feeling & $\begin{array}{c}\text { Number } \\
\text { of Dentists }\end{array}$ \\
\hline Dental work is physically demanding & \\
Yes & 8 \\
No & 4 \\
I am exhausted after work & \\
Mostly not & 4 \\
Mostly yes & 7 \\
Always & 1 \\
\hline
\end{tabular}

\subsection{Physical Demand and Feeling Exhausted After Work}

The dentists were asked if dental work was physically demanding. They were also asked to rate how exhausted they felt at the end of their working day. The results are shown in Table 3.

\subsection{Overtime Work}

The dentists were asked if they worked overtime. The results showed that most of them did not. Only
TABLE 4. Stress-Inducing Working Situations and Average Scores $(M \pm S D)$ of Dentists' Stress

\begin{tabular}{lc}
\hline Working Situation & Score \\
\hline Patients with physical limitations & $2.75 \pm 0.62$ \\
Patients do not cooperate & $3.17 \pm 0.83$ \\
$\begin{array}{l}\text { Patients dislike the treatment they } \\
\quad \text { are given }\end{array}$ & $3.50 \pm 1.24$ \\
Pain and anxiety in patients & $2.75 \pm 1.06$ \\
Cancelled or late appointments & $1.91 \pm 1.08$ \\
Difficult communication and & $2.08 \pm 1.31$ \\
$\quad$ interaction with staff & \\
Routine and dull work & $1.75 \pm 0.75$ \\
Patients do not accept treatment & $2.25 \pm 1.36$ \\
Difficult cases & $3.33 \pm 0.89$ \\
Keeping to schedule & $3.33 \pm 1.23$ \\
\hline
\end{tabular}

Notes. Scores: 1-no stress at all, 2-very low degree of stress; 3-low degree of stress; 4-moderate degree of stress; 5-high degree of stress; 6-very high degree of stress.

one out of the 12 dentists worked overtime, Monday to Friday, 12 times during the past month.

\subsection{Working Situations That Cause Stress}

Patients who disliked the treatment provided was the most stressful working situation among all dentists (Table 4).

\subsection{Part 2: Dentists' Attitude Towards Pd}

The dentists had used Pd for 8-36 months. The average time was $19.67 \pm 11.3$ months $(M \pm S D)$.

\subsubsection{How Often Did the Dentists Use Pd?}

Five dentists sometime used $\mathrm{Pd}$ and seven dentists always used it. In this study, use means that the dentist was concerned about (a) hardware, i.e., proprioceptive-derived tools and equipment, and (b) software, i.e., the working procedure, senses, feelings, and the relationship between him or her and the environment (derived from using Pd) while providing dental care. 


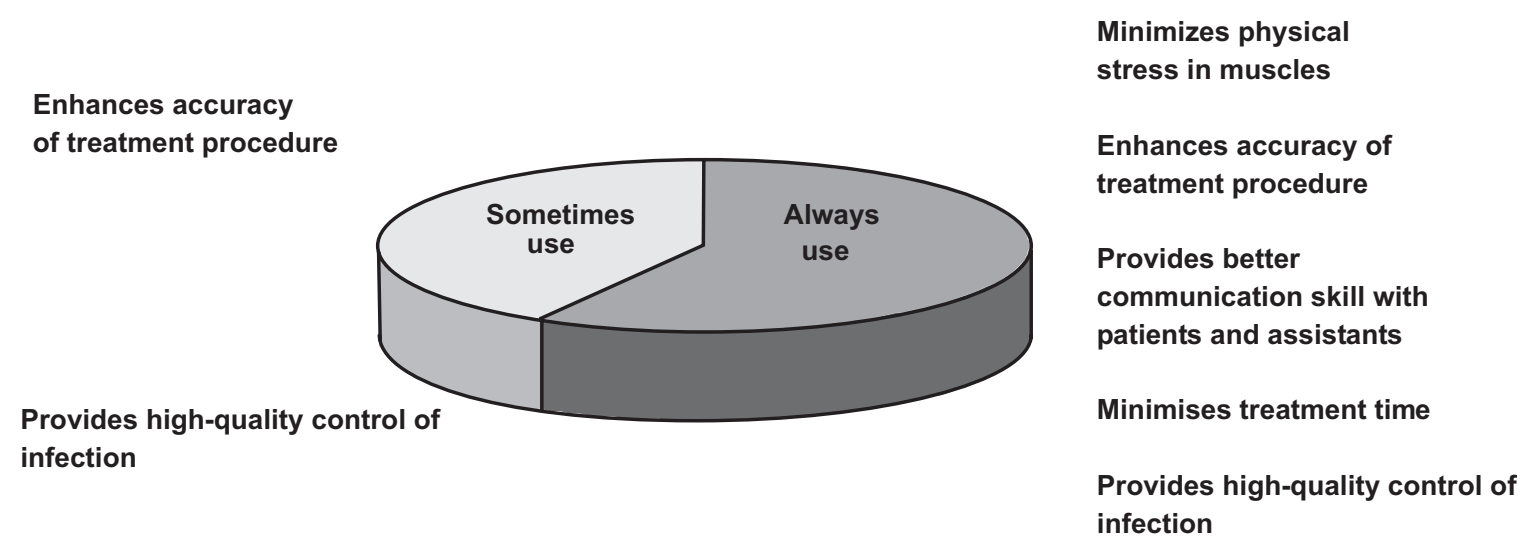

Figure 2. Reasons why dentists like Proprioceptive Derivation $(n=12)$

\subsubsection{Did the Dentists Like or Dislike Pd?}

Ten dentists liked Pd, with seven of them always using it. Only two dentists disliked Pd: both of them sometime used it.

\subsubsection{Why Did Dentists Like or Dislike Pd?}

The dentists who always used Pd said they liked it because it helped to reduce physical stress on the muscles, especially in the shoulder and back regions, enhance accuracy of the treatment procedure, enhance communication with patients and assistants, reduce treatment time, and increase high-quality control of infection (by minimizing finger-hand contact with the lamp and table). Three out of the five dentists who sometimes used Pd also mentioned that it helped to enhance the accuracy of the treatment procedure, and was good for infection control. Only two dentists did not like Pd, one dentist wrote that Pd on its own could not been applied to all types of dental practice. The other did not like or did not want to use it due to its complexity and difficulty (Figure 2).

\section{DISCUSSION}

This study showed that the majority of the dentists who used Pd liked it and reported improved work performance and reduced musculoskeletal load. However, few dentists participated in the study mainly because there is only one dental school in Thailand which uses Pd and this school has a limited number of staff members. In order to increase the number of participants in a future study, dental students who also use this concept should be included. Hence, it should be stressed that the results of this study are based on a small number of dentists using Pd; consequently it may not be appropriate to generalize those results to all users or to other participant groups. On the other hand, this study could have some benefit as one contribution regarding $\mathrm{Pd}$, as not many studies or much information has been available on this subject.

Dentists' working posture is a topic that many researchers have paid attention to because it is considered as a risk factor for musculoskeletal symptoms $[11,12,13,14]$. The results from this study showed that $100 \%$ of the dentists chose to work in a sitting posture. No one alternated their posture between sitting and standing, which might explain why some disliked Pd. Rundcrantz, Johnsson, and Moritz [11] reported that $95 \%$ of dentists worked in a sitting posture when working conventionally. Further, Finsen, Christensen, and Bakke [13] found that the majority $(82 \%)$ of dentists sat while working. Sitting continuously is considered a risk factor for low back pain because dentists who work in a sitting posture all the time had more severe back pain than those who alternated between sitting and standing [15]. An observation study of dentists using Pd would give more information on how they sit [16]. 
Interruptions or micropauses are presumed essential in decreasing or varying musculoskeletal load [17, 18]. However, only five dentists $(41.7 \%)$ had breaks of around $5 \mathrm{~min}$ between patients. Also, most dentists perceived dental work as physically demanding and felt exhausted at the end of the day. Ilmarinen, Suurnakki, Nygård, and Landau [19] reported that among 88 professions, the highest stress factor level was found in dentists, kitchen supervisors and physicians. Continuous sitting and no breaks are probably related to sitting in the same posture, and it is thus related to muscular discomfort, affecting the dentists' perception of dental work as a demanding job causing exhaustion after work.

Table 5 compares Pd users to dentists studied by Finsen, Christensen and Bakke [13]. There were differences in the average time spent on

TABLE 5. Time Spent on Dental Tasks in Two Studies

\begin{tabular}{|c|c|c|}
\hline \multirow[b]{2}{*}{ Dental Work Task } & \multicolumn{2}{|c|}{ Duration (min) } \\
\hline & $\begin{array}{l}\text { Current } \\
\text { Study }\end{array}$ & $\begin{array}{c}\text { Finsen, } \\
\text { Christensen, } \\
\text { \& Bakke's } \\
\text { Study [13] }\end{array}$ \\
\hline Dental examination & $9.0 \pm 7.7$ & $11.9 \pm 8.0$ \\
\hline Teeth cleaning & $24.1 \pm 5.9$ & $14.9 \pm 7.8$ \\
\hline Dental filling therapy & $24.6 \pm 5.2$ & $19.7 \pm 9.2$ \\
\hline $\begin{array}{l}\text { Crown and bridge } \\
\text { therapy }\end{array}$ & $42.0 \pm 21.0$ & $72.9 \pm 44.0$ \\
\hline Tooth extraction & $16.0 \pm 6.1$ & $15.9 \pm 9.6$ \\
\hline
\end{tabular}

dental work tasks. Pd users in this study spent less time on dental examination and crown and bridge therapy tasks. However, they spent more time on teeth cleaning and dental filling therapy tasks. These differences could be related to many factors such as dentists' skills, type of treatment and patients. Dentists who worked conventionally [13] spent less time on each dental work task compared to dentists in this study, which is contrary to expectations.
Fujita, Kawamoto, Kohmi, Onchi, Inoue, and Fujii [20] reported that skilled dentists have an advanced ability to have shorter cycle times when performing dental care under $\mathrm{Pd}$. An observation study with a video-based analysis [21] would help to clarify why dentists who used $\mathrm{Pd}$ in this study spent more time on each task. Also a paper-based task analysis [22] or actual time measurements on patient visits [23] could give more interesting information on the variability of working time in dental work tasks.

According to the responses, patients who disliked the given treatment was the most stressful situation for the dentists in this study. Gorter, Albrecht, Hoogstraten and Eijkman [24] reported similarly: dentists had the highest mean scores of work stress related to patient contacts and work contents items. Further from the present study, time management such as cancelled or late appointments and coping with difficult patients also caused stress. Similarly, Wilson, Coward, Capewell, Laidler, Rigby, and Shaw [25] found time management including running behind schedule, coping with difficult or uncooperative patients, and working constraints set by the National Health Service (NHS) as major job stressors among general dental practitioners. Furthermore, occupational stress in dental work also came from trying to sustain and build a practice, lack of career perspectives and having to cope with difficult patients [26, 27]. It seems that Pd does not help dentists in solving their problem with difficult patients and running behind schedule.

Interestingly, the stress score in solving problems in patients with physical limitations was lower among dentists in this study, compared to Cooper, Mallinger, and Kahn's study [26]. Pd, which includes a dental bed, provides easier accessibility to patients with limitations, such as the disabled and the elderly, which might be an advantage. Further, the special headrest might help the dentist to position the patient's head and to keep the patient's mouth in a predictable position regardless of the patient's height, and thus reduce stress in dentists. 
In this study, attitude meant a feeling or an opinion about Pd or a behaviour caused by that concept. The results showed that the main reason for using Pd was that it minimized physical stress in the muscles, especially in shoulder and back regions, enhanced the accuracy of treatment, provided better communication with patients and assistants, minimized treatment time, and minimized finger-hand contact with the light and chair. Interestingly, dentists who always or sometimes used the concept liked it because of two reasons: enhanced accuracy of treatment and high-quality control of infection. These results are in agreement with what is reported deriving from the skill program, the SATV system [10]. They claim that Pd equipment is required together with SATV. However, further studies need to be done to evaluate if SATV is sufficient for achieving high work quality and if SATV can be combined with conventional dental equipment or not.

Two dentists did not like Pd. One stated that, on its own, it did not cover all types of dental tasks and treatments. Additionally, one dentist did not want to use this concept due to its complexity and difficulty. These results might imply that $\mathrm{Pd}$ is not easy to learn because the two dentists were experienced in working conventionally prior to using Pd. Van Beer, Sittig, and Denier van der Gon [28] found that "the precision of proprioceptive localization of the hand in humans is based on three different sources of information: proprioceptive information about the left hand, proprioceptive information about the right hand, and visual information" (p. 367). Therefore, dentists may need more practice in order to get the right proprioceptive information before they can consciously perform dental care under Pd without difficulty. Consequently, further studies on the effect of the training period on dentists' performance may provide additional information to supplement that of the current study.

Hendrick [29] divided ergonomics technology and its application into four groups: (a) hardware ergonomics or human-machine interface technology, (b) environmental ergonomics or human-environment technology, (c) cognitive ergonomics or human-software technology, (d) macroergonomics or human-organization technology. It is also possible to discuss Pd and its ergonomics application in all aspects. In hardware ergonomics, it concerns dentist performance capabilities as applied to the design of the dental workstation (e.g., the dental bed). In its environmental ergonomics aspect, it concerns dentist capabilities and limitations caused by the stress imposed by environmental modalities (e.g., lighting, vibration). It can be seen that the dental workstation environment, according to this concept, tries to minimize the environmental stress on dentists' performance, enhance comfort for dentists and patients and enhance productivity. $\mathrm{Pd}$ shows its concern in the software ergonomics aspect as well, as to how dentists conceptualize and process information from proprioception to their practice by having a guideline on movements; the five movements, the ten-step protocol, and the SATV as a training system. Lastly, it also concerns macroergonomics as the results show that it benefits dentists by enhancing better communication between dentist and assistant, dentist and patient, and dentist and dentist. In conclusion, it seems that this concept has shown its importance in work design and its contribution in many aspects of ergonomics.

\section{CONCLUSIONS}

Pd seems to reduce the level of stress, but the continuous sitting posture is a risk for back pain in dentists, from the ergonomics point of view. $\mathrm{Pd}$, with a dental bed, can provide easy accessibility to patients with physical limitations and it results in a lower stress level among dentists. Most dentists who used Pd found it useful. However, further studies are needed regarding sitting postures, whether SATV program can be combined with conventional dental equipment, the training period needed to 
reach high work performance, and also the attitudes among the patients regarding the concept.

\section{REFERENCES}

1. Wieslander G, Norbäck D. Dental work. In: Brune D, Gerhardson G, Crockford GW, D'Auria D, editors. The workplace. Oslo, Norway: Scandinavian Science Publishers; 1997. vol. 2, p. 82-9.

2. Mangharam J, McGlothan J. Ergonomics and dentistry: a literature review. In: Murphy DC, editor. Ergonomics and the dental care worker. Washington, DC, USA: American Public Health Association; 1998. p. 25-75.

3. Pollack R. Dental office ergonomics: how to reduce stress factors and increase efficiency. J Can Dent Assoc 1996;62(6):508-10.

4. Finkbeiner BL. Four-handed dentistry: instrument transfer. J Contemp Dent Pract 2001;2(1):57-76.

5. Proprioceptive derivation [homepage of the Faculty of Dentistry, Thammasat University, Thailand]. Retrieved December 12, 2003 from: http://www.tu.ac.th/org/dentist/teach4.html. In Thai.

6. Dougherty M. Ergonomic principles are proprioceptive derived. Retrieved February 10, 2004 from: http://www.designbyfeel.com

7. Stedman's electronic medical dictionary [CD-ROM]. Baltimore, MD, USA: Lippincott William \& Wilkins; 1996.

8. Belenky MM. Human-centered ergonomics: proprioceptive pathway to occupational health and peak performance in dental practice. In: Murphy. DC, editor. Ergonomics and the dental care worker. Washington, DC, USA: American Public Health Association; 1998. p. 275-99.

9. Rucker LM, Sunell S. Ergonomic risk factors associated with clinical dentistry. J Calif Dent Assoc; 30(2): 139-48.
10. Terui Y, Iwao J, Taniguchi T. The effect of the SATV system. In: Seppälä P, Luopajärvi $\mathrm{T}$, Nygård $\mathrm{CH}$, Mattila $\mathrm{M}$, editors. Proceedings of the 13th Triennial Congress of the International Ergonomics Association, Tampere, Finland, 1997. Helsinki, Finland: Finnish Institute of Occupational Health; 1997. vol. 6, p. 244-6.

11. Rundcrantz BL, Johnsson B, Moritz U. Cervical pain and discomfort among dentists. Epidemiological, clinical and therapeutic aspects. Part 1. A survey of pain and discomfort. Swed Dent J 1990;14(2): 71-80.

12. Rohmert W, Bruder R. Ergonomic research and work design for the dentist. In: Singleton WT, Dirkx J, editors. Ergonomics, health and safety: perspectives for the nineties, Leuven, Belgium: Leuven University Press; 1991. p. 121-42.

13. Finsen L, Christensen H, Bakke $M$. Musculoskeletal disorders among dentists and variation in dental work. Appl Ergon 1998;29(2):119-25.

14. Pentikis J. Ergonomic Issues in dentistry, the U.S. Army perspective. In: Kumar S, editor. Advances in occupational ergonomics and safety 2 . Amsterdam, The Netherlands: IOS Press; 1998. p. 727-30.

15. Ratzon NZ, Yaros T, Mizlik A, Kanner T. Musculoskeletal symptoms among dentists in relation to work posture. Work 2000;15(3):153-8.

16. Chaikumarn M. Working posture and musculoskeletal discomfort in Thai dentists working with and without proprioceptive derivation concept [unpublished manuscript]; 2003.

17. Hagberg $M$, Hagberg C. Risks and prevention of musculoskeletal disorders among dentists. In: Brune, D.K, Edling C, editors. Occupational hazards among health professionals. Boca Raton, FL, USA: CRC Press; 1989. p. 323-32. 
18. Hagberg M, Wegmen DH. Prevalence rates and odds ratios of shoulder-neck diseases in different occupational groups. Br J Ind Med 1987;44(9):602-10.

19. Ilmarinen J, Suurnakki T, Nygård CH, Landau K. Classification of municipal occupations. Scand J Work Environ Health 1991;17(Suppl. 1):12-29.

20. Fujita M, Kawamoto M, Kohmi T, Onchi Y, Inoue M, Fujii B. Analysis of fine finger manipulation in dental practice. In: Seppälä $\mathrm{P}$, Luopajärvi $\mathrm{T}$, Nygård $\mathrm{CH}$, Mattila $\mathrm{M}$, editors. Proceedings of the 13th Triennial Congress of the International Ergonomics Association, Tampere, Finland, 1997. Helsinki, Finland: Finnish Institute of Occupational Health; 1997. vol. 6, p. 222-4.

21. Chaikumarn M. Variability in activities and postures during electronic assembly work assessed by video-based analysis system: a preliminary study [doctoral dissertation]. Luleå, Sweden: Luleå University of Technology; 2001.

22. Cameron CA, Beemsterboer PL, Johnson LA, Mislevy RJ, Steinberg LS, Breyer FJ. A cognitive task analysis for dental hygiene. J Dent Educ 2000;64(5):333-51.

23. Parker WA, Williams DL, Mayotte RV, James JJ, Mangelsdorff AD. A model for dental workload measurement. Am J Public Health 1982;72(9):1022-7.

24. Gorter RC, Albrecht G, Hoogstraten J, Eijkman MA. Measuring work stress among Dutch dentists. Int Dent J 1999;49(3): $144-52$.

25. Wilson RF, Coward PY, Capewell J, Laidler TL, Rigby AC, Shaw TJ. Perceived sources of occupational stress in general dental practitioners. Br Dent J 1998;184(10): 499-502.

26. Cooper CL, Mallinger $M$, Kahn $R$. Identifying sources of occupational stress among dentists. J Occup Psychol 1978; 51:227-34.

27. Gorter RC, Albrecht G, Hoogstraten J, Eijkman MA. Work place characteristics, work stress and burnout among Dutch dentists. Eur J Oral Sci 1998;106(6): 999-1005.

28. van Beers RJ, Sittig AC, Denier van der Gon JJ. The precision of proprioceptive position sense. Exp Brain Res 1998;122(4):367-77.

29. Hendrick HW. Ergonomics: an international perspective. In: Karwowski W, Marras WS, editors. Occupational ergonomics: principles of work design. Boca Raton, FL, USA: CRC Press; 2003. p. 1-3-1-5. 